\title{
Non-dimensional formulization of the critical hydraulic head difference for seepage failure of soil in front of sheet piles
}

\author{
T. Tanaka \\ Graduate School of Agricultural Science, Kobe University, Nada Kobe 657-8501, Japan
}

T. Miki

National Institute for Rural Engineering, NARO, Kannondai, Tsukuba Ibaraki 305-8609, Japan

\begin{abstract}
For the excavation of soil with a high ground water level within a cofferdam, seepage water flows through soil under a hydraulic head difference, $\mathrm{H}$, between up- and downstream sides of a sheet pile wall, and seepage failure is often a problem. Seepage failure sometimes occurs even when a cofferdam has been expertly constructed based on a current design method. There still remain various shortfalls in the theory of the seepage failure of soil. In this paper, the prismatic failure concept 2D (pfc-2D) is first presented, which is one of the conventional methods for designing against seepage failure. Next, the non-dimensional formulization of the critical hydraulic head difference against seepage failure, Hc, is discussed especially in two dimensions. When the prototype (real) and model (test) soils are geometrically similar, we discuss whether or not the non-dimensional formulization for the Hc value is possible. The possibilities of the non-dimensional formulizations of the Hc values in the case of the other flow conditions of 2DC, 3D, and AXS flows are also discussed.
\end{abstract}

\section{INTRODUCTION}

For the excavation of soil with a high ground water level within a cofferdam, seepage water flows through the soil under a hydraulic head difference, $H$, between the up- and downstream sides of a sheet pile wall, and seepage failure is often a problem. Seepage failure sometimes occurs even when a cofferdam has been expertly constructed based on a current design method. There still remain various shortfalls in the theory of the seepage failure of soil.

In experiments on 2D seepage failure of soil, the discharge, $Q_{15}$, increases linearly with increasing $H$ until a certain value, $H_{d}$, i.e., the hydraulic head difference at which the $H-Q_{15}$ curve diverts from linearity. $Q_{15}$ is the quantity of discharge which is translated to the value at 15 degrees Centigrade. At almost the same point of $H_{y}$ as $H_{d}$, the soil surface begins to settle on the upstream side and rise on the downstream side. $H_{y}$ is defined as the onset of soil deformation. The experimental results lead to the interesting conclusion that $H_{y}=H_{d}$ (Tanaka et al. 1999). Soil particles move from up- to downstream around the bottom tip of a sheet pile wall. As $H$ increases beyond $H_{d}, Q_{15}$ becomes larger with increasing $H$ more steeply than before, and the ground finally collapses at the hydraulic head difference at failure, $H_{f}$. The ground is subjected to irreversible damage and cannot be restored when $H$ increases beyond $H_{y}$. So, the head difference, $H$, must be designed to not be above $H_{y}$. The hydraulic head dif- ferences at deformation in an experiment, $H_{y}\left(=H_{d}\right)$, are nearly equal to the theoretical critical hydraulic head differences based on the prismatic failure concept (pfc), $H_{c}$, for the same cases.

In this paper, the prismatic failure concept (pfc) is first presented, which is one of the conventional methods for calculating soil excavation safety against seepage failure. The non-dimensional formulization of the critical hydraulic head difference against seepage failure, $H_{c}$, is discussed especially in two dimensions. In the discussion, the cases of 2DC, $3 \mathrm{D}$, and AXS flow conditions are also considered. If the non-dimensional formulization becomes available, the critical hydraulic head differences, $H_{c}$, can be precisely obtained from conditions of $2 \mathrm{D}, 2 \mathrm{DC}$, $3 \mathrm{D}$, and AXS flows, as well as information about the total depth of soil, penetration depth of sheet piles, and plane shapes of a cofferdam without performing complex calculations.

\section{FLOW CONDITIONS}

It has been found that the aspects of seepage failure of soil (with a high ground water level) are markedly influenced by the flow conditions (Tanaka et al. 2009). When excavating a large area with a high ground water level, as shown in Figure 1, the seepage failure of soil in front of a sheet pile wall is a problem in two dimensions, which is designated as 
two-dimensional flow (2D flow). In the excavation of soil between double sheet pile walls, as shown in Figure 2, seepage water concentrates into the soil two-dimensionally from the outside, which is called two-dimensional concentrated flow (2DC flow). The 2DC flow of water lowers the safety factor regarding the seepage failure of soil. When the longitudinal length of the double sheet pile walls is small, as shown in Figure 3, seepage flow concentrates threedimensionally into soil surrounded by a rectangular wall, which is called three-dimensional concentrated flow (3D flow). The 3D flow condition further lowers the safety factor for seepage failure. An axisymmetric seepage flow through soil within a cylindrical wall is often used to model such a three-dimensional flow (Nagai et al. 2016). The cylindrical wall condition brings about axisymmetrically concentrated flow (AXS flow), as shown in Figure 4. The seepage flow during the excavation of soil in a cylindrical shaft with a high ground water level is just the condition of the AXS flow.

In this paper, we discuss the non-dimensional formulization of the critical hydraulic head difference for the seepage failure issues of soil in two dimensions. The method of calculating stability against the seepage failure of soil -the prismatic failure concept in two dimensions (pfc-2D)- is presented, and the theory of non-dimensional formulization of the critical hydraulic head difference is discussed. The pfc-3D and pfc-AXS are not made reference to, but whether or not the non-dimensional formulization is appropriate is discussed regarding all flow conditions: 2D, 2DC, 3D, and AXS flows.

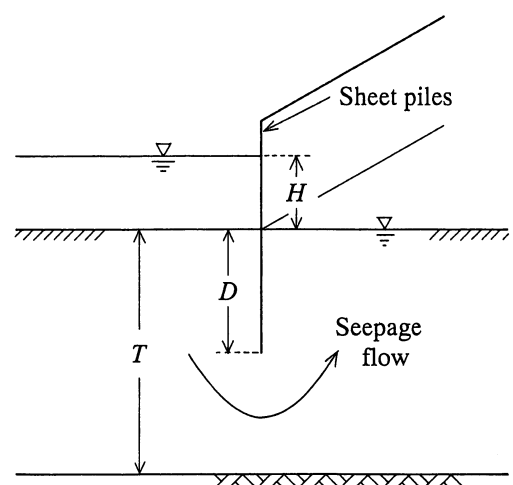

Figure 1. Two dimensional flow (2D flow).

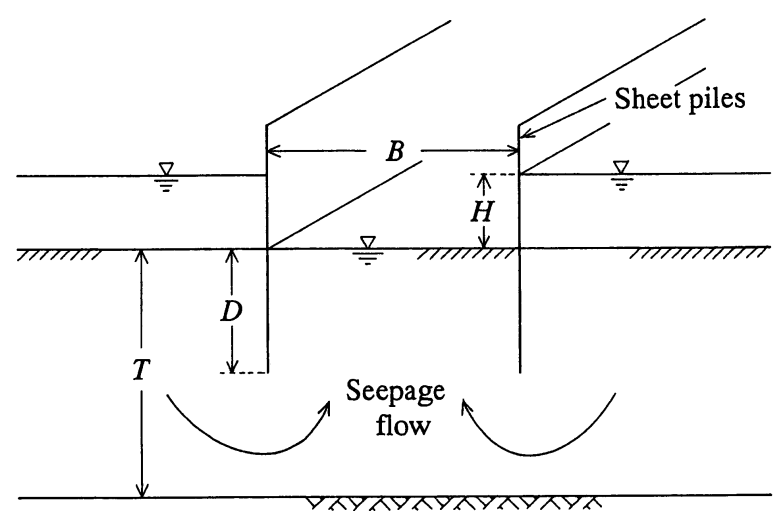

Figure 2. Two-dimensional concentrated flow (2DC flow).

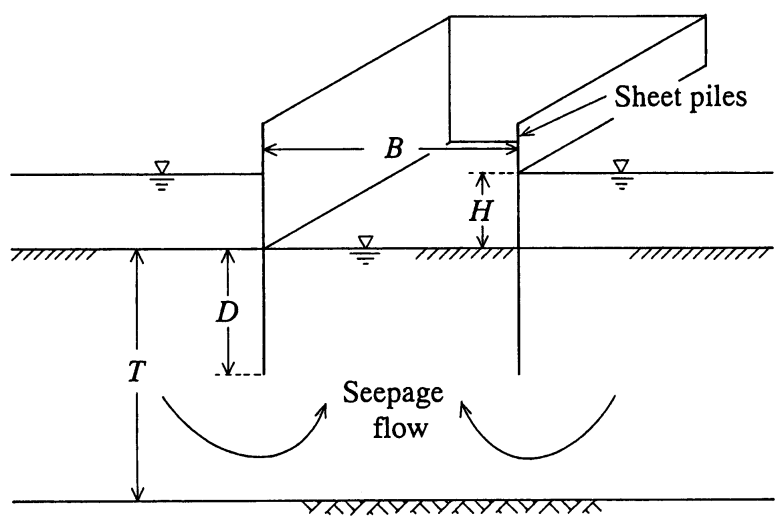

Figure 3. Three-dimensional flow (3D flow).

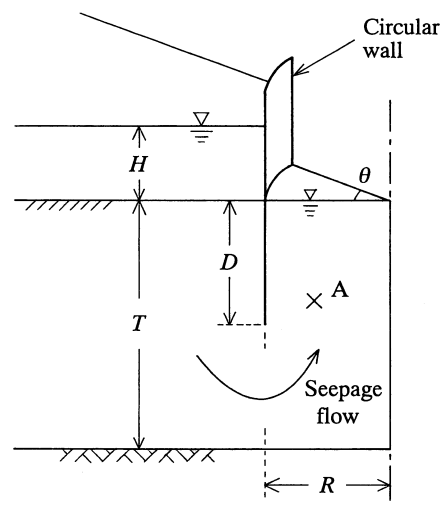

Figure 4. Axisymmetric flow (AXS flow).

\section{PRISMATIC FAILURE CONCEPT IN TWO DIMENSIONS}

The prismatic failure concept (Tanaka 1966, Tanaka \& Verruijt 1999) is an extension of Terzaghi's method (Terzaghi 1943). In the prismatic failure concept, we assume that the body of soil lifted by seepage water has the shape of a prism with a certain height and width adjoining a sheet pile wall. Let us consider, for instance, the prism OABC in homogeneous soil, as shown in Figure 5. As we can see in Figure 6, the rise of the prism is resisted by the submerged weight, $W$, and frictions, $F_{L}$ and $F_{R}$, on the left and right sides, respectively. The safety factor $F_{s}$ with respect to the rise of the prism, which is subjected to the total excess pore water pressure on its base, $U_{e}$, is given as:

$F_{s}=\frac{W^{\prime}+F_{L}+F_{R}}{U_{e}}$.

The values on the right side in Eq.(1) are expressed as:

$$
\begin{aligned}
& W^{\prime}=\gamma^{\prime} b l, \\
& U_{e}=\left.\int_{x_{0}}^{x_{0}+b} u_{e}\right|_{z=z_{0}} d x, \\
& F_{L}=\left.\int_{z_{0}}^{z_{0}+l} \sigma_{x}^{\prime}\right|_{x=x_{0}} \tan \delta_{1} d z, \text { and }
\end{aligned}
$$


$F_{R}=\left.\int_{z_{0}}^{z_{0}+l} \sigma_{x}{ }^{\prime}\right|_{x=x_{0}+b} \tan \delta_{2} d z$

in which $\gamma^{\prime}$ is the buoyant unit weight of sand, $l$ is the height of a prism, $x_{0}$ and $z_{0}$ are the coordinates of the bottom left point $\mathrm{O}$ of the prism (see Fig. 6), $u_{e}$ is the excess pore water pressure at the bottom of the prism, $\sigma_{x}$ ' is the horizontal effective stress, $\delta_{1}$ is the angle of friction on the left-hand side between the sheet pile wall and sand, and $\delta_{2}$ is the angle of friction on the right-hand side between sands.

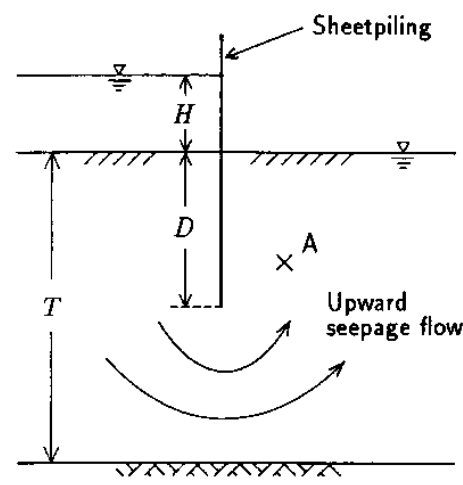

(a) Cofferdam

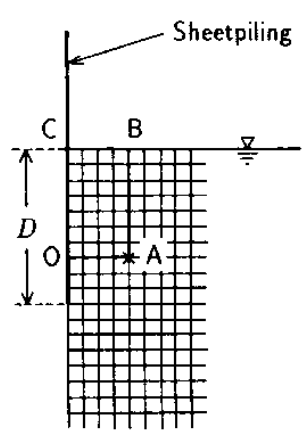

(b) Prism
Figure 5. Soil in front of sheet piles.

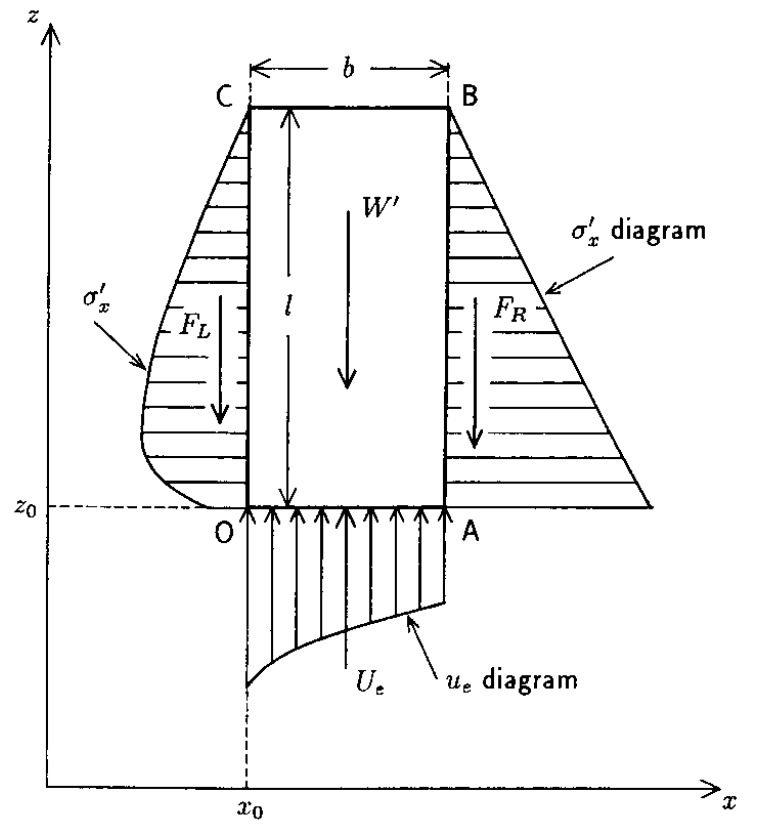

Figure 6. Forces acting on a prism.

For the hydraulic head difference $(H)$ between upand downstream sides, safety factors $\left(F_{s}\right)$ are calculated for all of the prisms in front of sheet piles. The safety factor $\left(F_{s}\right)$ takes the minimum value $\left(F_{s} \min \right)$ for a certain prism among all of the prisms. The calculation is iterated for another hydraulic head difference, $H$, until the condition whereby $F_{s}$ min becomes nearly equal to 1.0 . The head difference $H=H_{c}$ at which the condition $F_{s \text { min }}=1.0$ is applied is defined as the critical hydraulic head difference. This approach is based on the minimum criterion by Kälin (1977). The prism with a value of $F_{s \min }=1.0$ among all of the prisms for $H=H_{c}$ is defined as the critical prism. We could say that the critical prism is sepa- rated from the underlying soil at its base when $H$ exceeds $H_{c}$. Safety factors using the prismatic failure concept when considering friction, $F_{P F}$, are discussed here. The above approach of pfc was explained only for 2 dimensions, but pfc-3D (Tanaka et al. 2012) and pfc-AXS (Tanaka et al. 2000) can also be defined in the same way.

\section{NON-DIMENSIONAL FORMULIZATION OF THE CRITICAL HYDRAULIC HEAD DIFFERENCE}

Let us consider the non-dimensional formulization of the critical hydraulic head difference against the seepage failure of soil in two dimensions, as shown in Figure 1. We assume that the real and test soils in two dimensions are geometrically similar, consisting of the same material (sand) and under the same conditions. Here, the real soil is referred to as the prototype, and the test soil as the model.

\subsection{Theory of non-dimensional formulization based on $p f c-2 D$}

Let us consider the two-dimensional $x-z$ coordinate system. The basic equation of seepage flow through soil with an anisotropic permeability is given as:

$k_{x x} \frac{\partial^{2} h}{\partial x^{2}}+2 k_{x z} \frac{\partial^{2} h}{\partial x \partial z}+k_{z z} \frac{\partial^{2} h}{\partial z^{2}}=0$

where $h$ is the total hydraulic head, and $k_{x x}, k_{z z}$, and $k_{x z}$ are the coefficients of permeability tensor in two dimensions. Referring to the ratio of corresponding lengths of the model and prototype as $l_{r}$, we have the relationship:

$L_{\mathrm{mod}}=l_{r} L_{\mathrm{pro}}$

where $L$ is an arbitrary length corresponding to each soil, the subscript "mod" represents model or test soil, "pro" is the prototype or real soil, and the scale $l_{r}$ is the same for the horizontal $x$ and vertical $z$ directions. So the coordinates $x_{\bmod }$ and $z_{\bmod }$ in test soil correspond to $x_{\text {pro }}$ and $z_{\text {pro }}$ in the real soil as follows, respectively:

$x_{\text {mod }}=l_{r} x_{\text {pro }}$,

$z_{\text {mod }}=l_{r} z_{\text {pro }}$.

In the case of a homogeneous soil, excess pore water pressure (expressed in water head), $h_{e}$, on the bottom of a prism is represented as follows, because the partial differential equation (Eq.(6)) is linear with respect to the water head, $h$ :

$h_{e \bmod }=l_{r} h_{e \text { pro }}$.

The excess pore water pressure, $u_{e}$, means a net pressure which actually causes a seepage flow, and is 
represented as follows, using the value expressed in the water head, $h_{e}$ :

$u_{e}=\gamma_{w} h_{e}=\rho_{w} g h_{e}$,

so the value of $u_{e}$ is represented as, using Eqs.(8) and (9):

$u_{e \bmod }=l_{r} u_{e \text { pro }}$

in which $\gamma_{w}$ is the unit weight of water, $\rho_{w}$ the density of water, and $g$ the acceleration of gravity. A pressure acts on an area, so it must be represented as a square of the length ratio, $l_{r}^{2}$, but is given as $l_{r}$ in Eq.(10). This is because we consider a unit depth (perpendicular to the page), and one side of the area is a unit depth. The same can be said of the stresses discussed below (see Eqs.(12) and (17)). The vertical effective stress at point $z, \sigma_{z}$ ', is calculated by:

$\sigma_{z}^{\prime}=\gamma^{\prime} z-u_{e}$

and the following relationship is obtained for the corresponding points from Eqs.(7c), (10), and (11):

$\sigma_{z}{ }^{\prime}$ mod $=\gamma_{\text {mod }}^{\prime} z_{\text {mod }}-u_{e \text { mod }}=l_{r}\left(\gamma_{\text {pro }}^{\prime} z_{\text {pro }}-u_{e \text { pro }}\right)=l_{r} \sigma_{z}{ }^{\prime}$ pro.

The real and test soils consist of the same sand, under the same conditions, and under the same gravitation, so the following relationships are applied:

$\gamma^{\prime}=\gamma_{\text {mod }}^{\prime}=\gamma_{\text {pro }}^{\prime}$,

$\gamma^{\prime}=\rho^{\prime} g, \gamma_{\text {mod }}^{\prime}=\rho_{\text {mod }}^{\prime} g, \quad \gamma_{\text {pro }}^{\prime}=\rho_{\text {pro }}^{\prime} g$, and

$\rho^{\prime}=\rho_{\text {mod }}^{\prime}=\rho_{\text {pro }}^{\prime}$

where $\gamma^{\prime}$ and $\rho$ ' are the unit weight and density of soil, respectively.

The horizontal effective stress $\sigma_{x}$ ' is given as, using the coefficient of a lateral earth pressure $K$ :

$\sigma_{x}^{\prime}=K \sigma_{z}^{\prime}$

The coefficients of earth pressure $K$ values are given as $K_{p}$ for the passive state and $K_{0}$ for the state at rest according to the states of soil. These values are given as follows, by Rankin and Jáky, respectively:

$K_{p}=\frac{1-\sin \phi^{\prime}}{1+\sin \phi^{\prime}}$, and

$K_{0}=1-\sin \phi^{\prime}$

in which $\phi^{\prime}$ is the internal friction angle with respect to the effective stress. Assuming that the real and test soils are the same and under the same conditions (or $\phi$ ' is the same for the two soils), the relation:

$K_{\text {mod }}=K_{\text {pro }}$

is applied. From Eqs.(12), (14), and (16), we can have:

$\sigma_{x}^{\prime}{ }_{\text {mod }}=l_{r} \sigma_{x}^{\prime}{ }^{\prime}$ pro.
Now, we consider Eq.(1) for calculating the safety factor against seepage failure of the real and test soils. For the corresponding buoyant weights of prisms $W^{\prime}$ mod and $W^{\prime}$ pro are related to each other:

$W_{\text {mod }}^{\prime}=l_{r}^{2} W_{\text {pro }}^{\prime}$.

A buoyant weight of a prism acts in a volume, so it must be represented as the cube of the length ratio, $l_{r}^{3}$, but is given as $l_{r}^{2}$ in Eq.(18). This is because we consider a unit depth (perpendicular to the page), and one side of the prism concerned is a unit depth. The frictional forces acting on the left and right-hand sides of the prism, $F_{L}$ and $F_{R}$, respectively, are the integrals of $\left(\sigma_{x}{ }^{\prime} \tan \delta\right)$ along those sides. If the angles of friction on the left and right-hand sides, $\delta_{1}$ (between the sheet pile wall and sand), and on the righthand side, $\delta_{2}$ (between sands), are the same for the real and test soils, respectively, the following equations are obtained, using Eqs.(4), (5), (12), and (14):

$F_{L \text { mod }}=l_{r}^{2} F_{L \text { pro }}$, and

$F_{R \text { mod }}=l_{r}^{2} F_{R \text { pro }}$

in which $\delta_{1}=2 \phi^{\prime} / 3$ and $\delta_{2}=\phi^{\prime}$ are used in our calculation (Yamaguchi 1976, Soil Engineering Library 1975). Next, we consider the total excess pore water pressure acting on the prism, $U_{e}$. The value of $U_{e}$ is an integral of the excess pore water pressure, $u_{e}$, on the bottom of the prism, so the following relationship is obtained, using Eqs.(3), (7b), and (10):

$U_{e \text { mod }}=l_{r}^{2} U_{e \text { pro }}$.

For the corresponding prisms of the real and test soils, safety factors against seepage failure are related to each other:

$F_{s \bmod }=\frac{W_{\mathrm{pro}}^{\prime}+F_{L \mathrm{mod}}+F_{R \bmod }}{U_{e \bmod }}=\frac{l_{r}^{2}\left(W_{\mathrm{pro}}^{\prime}+F_{L \mathrm{pro}}+F_{R \mathrm{pro}}\right)}{l_{r}^{2} U_{e \text { pro }}}=F_{s \text { pro }}$

Thus, the safety factors against seepage failure are the same for the real and test soils. The critical prisms, which give a safety factor $\left(F_{s}\right)$ a minimum value $\left(F_{s}\right.$ min $)$ and 1.0 simultaneously among all prisms, are located at the same position for both soils.

As for the critical hydraulic head difference, we have:

$H_{c \text { mod }}=l_{r} H_{c \text { pro }}$.

For the total depth of soil, $T$, and the penetration depth of sheet piles, $D$, we have the relations, using Eq.(7a):

$T_{\text {mod }}=l_{r} T_{\text {pro }}, D_{\text {mod }}=l_{r} D_{\text {pro }}$,

so, the relationships $D_{\text {mod }} / T_{\text {mod }}=D_{\text {pro }} / T_{\text {pro }}, H_{\text {mod }} / D_{\text {mod }}$ $=H_{\text {pro }} / D_{\text {pro }}$, and $H_{\text {mod }} / T_{\text {mod }}=H_{\text {pro }} / T_{\text {pro }}$ are true, and the non-dimensional values of $D / T, H / D$, and $H / T$ 
are the same for the two soils. From a theoretical point of view, the relationship between a nondimensional value, $D / T$, and normalized values $H \gamma_{w} / D \gamma^{\prime}$ and $H \gamma_{w} / T \gamma^{\prime}$ must be the same for the real and model soils. The normalized values of $H \gamma_{w} / D \gamma^{\prime}$ and $H \gamma_{w} / T \gamma^{\prime}$ are given as the non-dimensional values $H / D$ and $H / T$ divided by another non-dimensional value, $\gamma^{\prime} / \gamma_{w}$, which is the critical hydraulic gradient of soil subjected to an upward seepage flow. We should be aware that the relation $\gamma_{\text {mod }}^{\prime} / \gamma_{w}=\gamma_{\text {pro }}^{\prime} / \gamma_{w}$ is used to obtain the equations above.

The aforesaid results will be obtained with the following:

(1) We consider the real and test soils in two dimensions which are geometrically similar, consisting of the same material (sand) and under the same conditions.

(2) The partial differential equation (6) is linear with respect to the water head, $h$.

(3) The equations (7c), (10), (11) and (14) for representing respective pressures (and effective stresses) acting on the prisms of the test and real soils are linear to the scale of a length, $l_{r}$, because one side of the prism concerned is a unit depth (perpendicular to the page).

\subsection{Theoretical consideration about buoyant unit weight of soil $\gamma$ '}

Here, we consider the case where the real and test soils have the same size (or $l_{r}=1.0$ ) but different buoyant unit weights, i.e.:

$L_{\text {mod }}=L_{\text {pro }}$,

$x_{\text {mod }}=x_{\text {pro }}$,

$z_{\text {mod }}=z_{\text {pro }}$, and

$\gamma_{\text {mod }}^{\prime}=\gamma_{r}^{\prime} \gamma_{\text {pro }}^{\prime}$

in which $\gamma_{r}^{\prime}$ is the ratio of corresponding buoyant unit weights of the model and prototype, $\gamma_{r}^{\prime}=\gamma_{\text {mod }}^{\prime}$ $/ \gamma_{\text {pro. }}^{\prime}$

As a matter of convenience, we suppose that the real soil has $\gamma_{\text {pro }}^{\prime}$ and the test soil has a different value, $\gamma_{\text {mod }}^{\prime}\left(=\gamma_{r}^{\prime} \gamma_{\text {pro }}^{\prime}\right)$. As for the model soil, we have the following relations from Eqs.(2) and (11), using Eqs.(25c) and (26):

$W_{\text {mod }}^{\prime}=\gamma_{\text {mod }}^{\prime} b l=\gamma_{r}^{\prime} \gamma_{\text {pro }}^{\prime} b l=\gamma_{r}^{\prime} W_{\text {pro }}^{\prime}$,

$\sigma_{z}^{\prime}{ }_{\text {mod }}=\gamma_{\text {mod }}^{\prime} z_{\text {mod }}-u_{e \bmod }=\gamma_{r}{ }^{\prime} \gamma_{\text {pro }}^{\prime} z_{\text {pro }}-u_{e \bmod }$.

Assuming that the values of $h$ and $\gamma^{\prime}$ are independent from each other, the total hydraulic head, $h$, is thought to be given as follows for the corresponding (or the same) points in two soils:

$h_{e \bmod }=\gamma_{r}^{\prime} h_{e \text { pro }}$.

From Eqs.(9) and (29), we can obtain: $u_{e \bmod }=\gamma_{r}^{\prime} u_{e \text { pro }}$.

The value of $\sigma_{z}$ 'mod is derived as follows, using Eqs.(28) and (30):

$$
\begin{gathered}
\sigma_{z}^{\prime}{ }_{\text {mod }}=\gamma_{r}^{\prime} \gamma_{\text {pro }}^{\prime} z_{\text {pro }}-u_{e \text { mod }}=\gamma_{r}^{\prime} \gamma_{\text {pro }}^{\prime} z_{\text {pro }}-\gamma_{r}^{\prime} u_{e \text { pro }}= \\
\gamma_{r}^{\prime}\left(\gamma_{\text {pro }}^{\prime} z_{\text {pro }}-u_{e \text { pro }}\right)=\gamma_{r}^{\prime} \sigma_{z}^{\prime} \text { pro. }
\end{gathered}
$$

The frictional forces acting on the left and righthand sides of the prism, $F_{L}$ and $F_{R}$, respectively, are the integrals of $\left(\sigma_{x}{ }^{\prime} \tan \delta\right)$ along those sides. If the coefficients of friction are the same for real and test soils, the following equations are obtained from Eqs.(4), (5), (14), and (31), in a similar way as in Sec.4.1:

$F_{L \text { mod }}=\gamma_{r}^{\prime} F_{L \text { pro }}$, and

$F_{R \bmod }=\gamma_{r}^{\prime} F_{R \text { pro }}$.

The $U_{e}$ value is an integral of the excess pore water pressure $u_{e}$ on the bottom of the prism, so the following relationship is obtained, using Eqs.(3), (25b), and (30):

$U_{e \bmod }=\gamma_{r}{ }^{\prime} U_{e \text { pro }}$.

For the corresponding prisms of the real and test soils, safety factors against seepage failure are related to each other:

$$
F_{s \bmod }=\frac{W_{\text {mod }}^{\prime}+F_{L \text { mod }}+F_{R \bmod }}{U_{e \bmod }}=\frac{\gamma_{r}^{\prime}\left(W_{\text {pro }}^{\prime}+F_{L \text { pro }}+F_{R \text { pro }}\right)}{\gamma_{r}^{\prime} U_{e \text { pro }}}=F_{s \text { pro }}
$$

Thus, the safety factors against seepage failure are the same for the real and test soils, in which the buoyant unit weights $\left(\gamma^{\prime}\right)$ are different. The critical prism, which gives a safety factor $\left(F_{s}\right)$ a minimum value $\left(F_{S} \min \right)$ and 1.0 simultaneously among all of the prisms, is located at the same position for each soil.

As for the critical hydraulic head difference, $H_{c}$, we have:

$H_{c \bmod }=\gamma_{r}^{\prime} H_{c \text { pro. }}$.

So, the values of $H / D$ and $H / T$ are not the same for the two soils, respectively, in this case. The nondimensional and normalized values $H_{c} \gamma_{w} / T \gamma^{\prime}$ and $H_{c} \gamma_{w} / D \gamma$ ' are given as follows for the model and real soils, respectively:

$$
\begin{aligned}
& \frac{H_{c \text { mod }} \gamma_{w}}{T_{\text {mod }} \gamma_{\text {mod }}^{\prime}}=\frac{\gamma_{r}^{\prime} H_{c \text { pro }} \gamma_{w}}{T_{\text {pro }} \gamma_{r}^{\prime} \gamma_{\text {pro }}^{\prime}}=\frac{H_{c \text { pro }} \gamma_{w}}{T_{\text {pro }} \gamma_{\text {pro }}^{\prime}}, \text { and } \\
& \frac{H_{c \text { mod }} \gamma_{w}}{D_{\text {mod }} \gamma_{\text {mod }}^{\prime}}=\frac{\gamma_{r}^{\prime} H_{c \text { pro }} \gamma_{w}}{D_{\text {pro }} \gamma_{r}^{\prime} \gamma_{\text {pro }}^{\prime}}=\frac{H_{c \text { pro }} \gamma_{w}}{D_{\text {pro }} \gamma_{\text {pro }}^{\prime}} .
\end{aligned}
$$




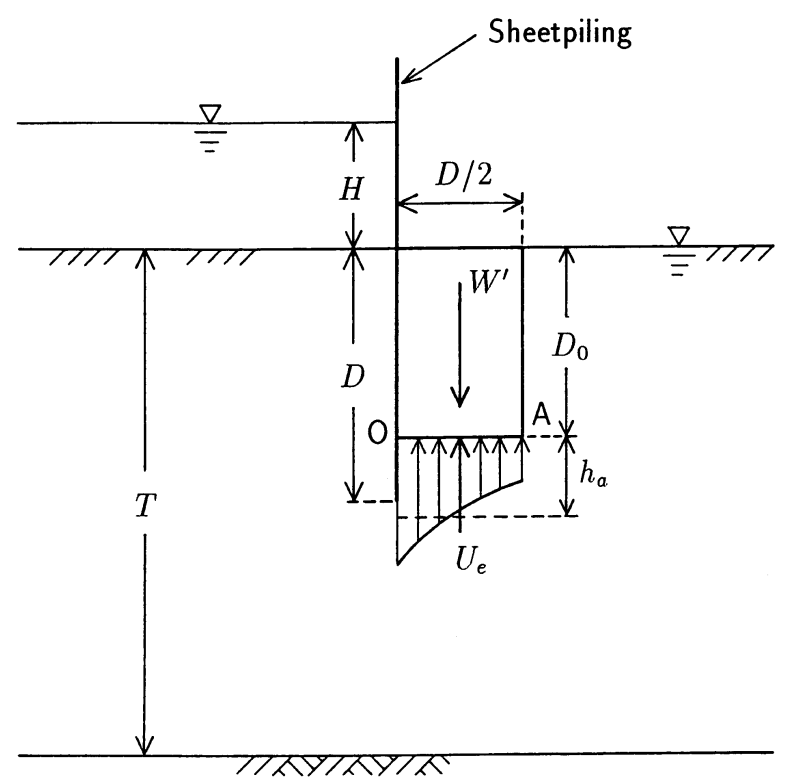

Figure 7. Terzaghi's method.

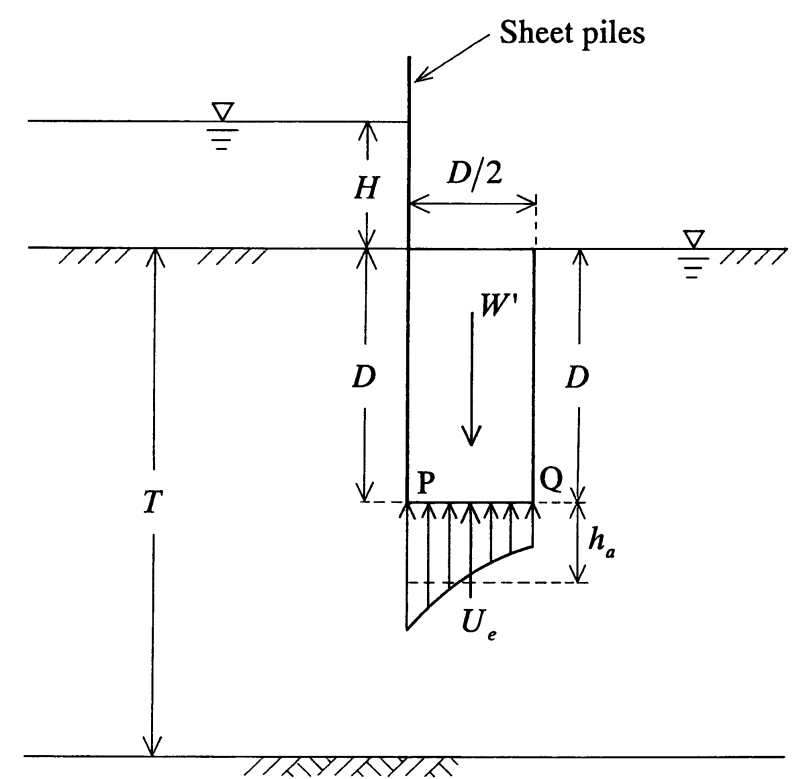

Figure 8. Terzaghi's prism of failure in the case of homogeneous soil.

Values of $H_{c} \gamma_{w} / T \gamma^{\prime}$ and $H_{c} \gamma_{w} / D \gamma^{\prime}$ are the same for the two soils, respectively. Therefore, these values are thought to be appropriate as non-dimensional and normalized values. When deriving these equations in this Section 4.2, we use the relations of $T_{\bmod }=T_{\text {pro }}$ and $D_{\text {mod }}=D_{\text {pro }}$.

\subsection{Formulization of $H_{c}$ based on Terzaghi's method}

In this section, why we use the ' $D / T-H_{c} \gamma_{w} / T \gamma$ ' curves" for the seepage failure issues of soil in front of sheet piles is discussed. Let us first consider Terzaghi's method, which is said to effectively represent the experimental results. Terzaghi (1943) assumed, from experimental evidence, that the body of soil adjacent to the sheet piles with a width $D / 2$ and depth $D_{0}$, as shown in Figure 7, is lifted up by seepage water and collapses. The rise of the prism is resisted by its weight and side friction. It is assumed that, at the instant of failure, the effective stress on the sides of the prism and the corresponding frictional resistance are practically zero. Therefore, the prism rises as soon as the total excess pore water pressure, $U_{e}$, on its base, OA, becomes equal to the submerged weight of the prism, $W^{\prime}$. At a given hydraulic head, $H$, the safety factor against the rise of a prism, $F_{s}$, depends on the value of $D_{0}$. The minimum value of $F_{s}$ among all of the $D_{0}$ values is the safety factor of the soil (Terzaghi 1943). For the single sheet pile wall condition, as illustrated in Figure 8, an investigation revealed that the bottom of the critical prism coincides with the lower edge of the sheet piles (Terzaghi \& Peck 1948), and the $F_{s}$ value is represented as:

$$
F_{s}=\frac{W^{\prime}}{U_{e}}=\frac{\gamma^{\prime} D}{h_{a} \gamma_{w}}=\frac{\gamma^{\prime} D}{C_{0} H \gamma_{w}},
$$

where $W^{\prime}$ is the buoyant weight of the prism, $U_{e}$ is the total excess pore water pressure acting on the base of the prism, $\gamma^{\prime}$ is the buoyant unit weight of soil, $\gamma_{w}$ is the unit weight of water, $h_{a}\left(=C_{0} H\right)$ is an average value of excess pore water pressure head acting on the bottom of the prism, and $C_{0}$ is a value depending on the hydraulic, geometric, and soil conditions.

Substituting $F_{s}=1.0$ into Eq.(39), we find the critical hydraulic head difference by Terzaghi $H_{c}$ as:

$$
H_{c}=\frac{\gamma^{\prime} D}{C_{0} \gamma_{w}}=\frac{1}{C_{0}} \frac{\gamma^{\prime} D}{\gamma_{w}} \text {. }
$$

It was found that Eq.(40) effectively represents the experimental results (Tanaka 1996, Tanaka \& Verruijt 1999). So, Eq.(40) can be utilized for representing the non-dimensional formulization of the $H_{c}$ value.

\subsubsection{For soil of an infinite depth}

Here, we consider the case where the lower impervious boundary is very deep, i.e., the soil is of an infinite depth. It means that the total depth of soil has no influence on seepage failure phenomena. In this case, dividing both sides of Eq.(40) by $D$ for nondimensional, and by $\gamma^{\prime} / \gamma_{w}$ for normalized:

$$
\frac{\gamma_{w} H_{c}}{D \gamma^{\prime}}=\frac{1}{C_{0}}=\text { Const. }
$$

where $C_{0}$ is a constant. Eq.(41) shows that, for 2D soil of an infinite depth, only one non-dimensional and normalized value, $H_{c} \gamma_{w} / D \gamma^{\prime}$ (= Const.), is given. 


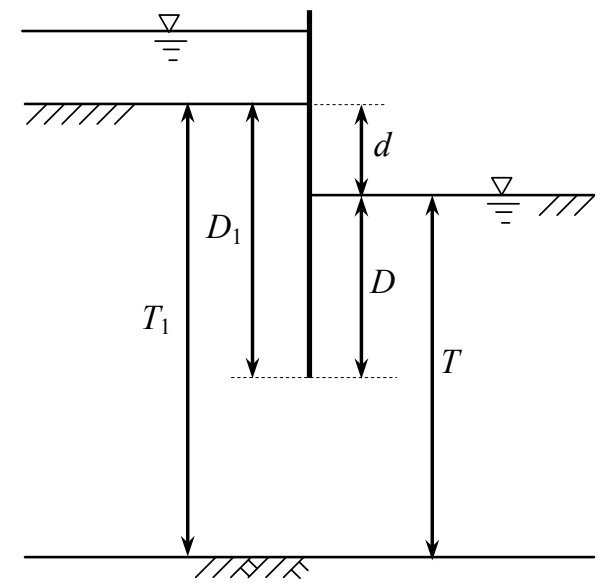

Figure 9. Notation of $2 \mathrm{D}$ flow for excavation case

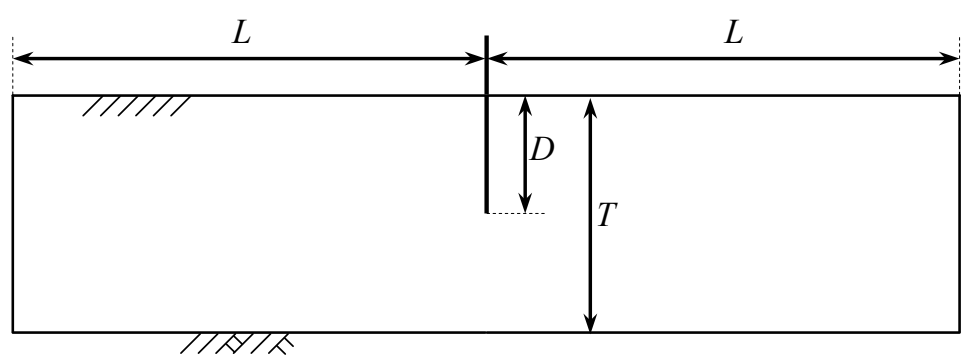

Figure 10. 2D flow

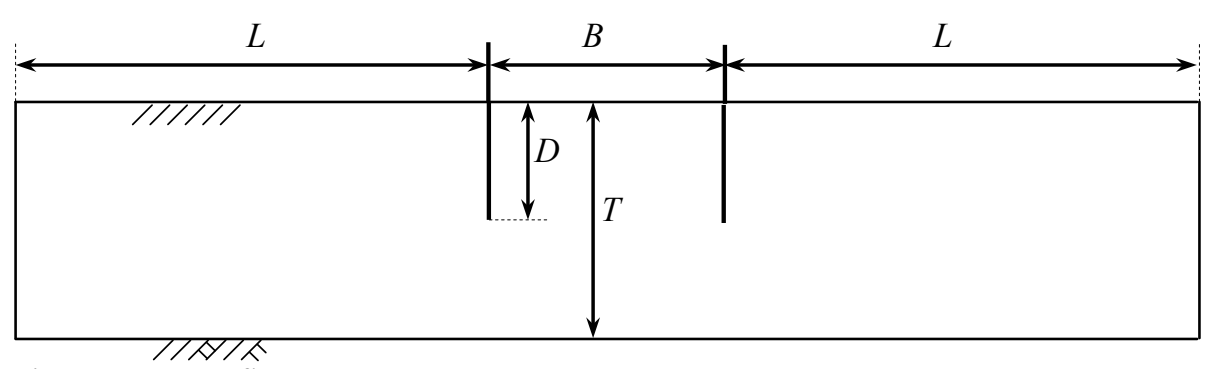

Figure 11. 2DC flow

\subsubsection{For soil of a finite depth}

Here, we consider the case where the lower impervious boundary is located at a finite depth, i.e., the soil is of a finite depth. It means that the seepage flow is restricted to the part of soil around/under sheet piles. In this case, it has been found that the total depth of soil has a marked effect on seepage failure phenomena (Tanaka \& Verruijt 1999). In this case, dividing both sides of Eq.(40) by $T$ for non-dimensional, and by $\gamma^{\prime} / \gamma_{w}$ for normalization:

$$
\frac{\gamma_{w} H_{c}}{T \gamma^{\prime}}=\frac{1}{C_{0}} \frac{D}{T}
$$

where $C_{0}$ is a function of $D / T$. It can be found from Eq.(42) that for 2D soil of a finite depth, a nondimensional and normalized relationship (or curve), $D / T-H_{c} \gamma_{w} / T \gamma^{\prime}$, is obtained.

\subsubsection{Non-dimensional formulization}

The prismatic failure concept (pfc) is an extension of Terzaghi's method, so the same relationships (or $H_{c} \gamma_{w} / D \gamma^{\prime}=$ Const. for an infinite depth and $D / T-$ $H_{c} \gamma_{w} / T \gamma$ ' curve for a finite depth) can be used for representing analytical results based on pfc.
From a physical point of view, the nondimensional value $D / T$ is the penetration ratio of a sheet pile wall, and $H_{c} \gamma_{w} / D \gamma^{\prime}$ and $H_{c} \gamma_{w} / T \gamma^{\prime}$ are the non-dimensional values of $H_{c} / D$ and $H_{c} / T$, which are normalized by another non-dimensional value, $\gamma^{\prime} / \gamma_{w}$, respectively. The value of $\gamma^{\prime} / \gamma_{w}$ is a critical hydraulic gradient against boiling of a one-dimensional soil subjected to an upward seepage flow.

The above discussion is only true for a noexcavation case $(d=0)$, and the excavation ratio $d /(D+d)$ is also added as a new parameter for the case of excavation $(d>0)$, as shown in Figure 9. The value of $d$ is the excavation depth on the downstream side.

In the above discussion, only a discussion on the 2D flow case (see Fig. 10) was made. In the case of 2DC and AXS flow (see Figs. 11 and 13), the new parameters of the ratio of a half breadth of double sheet piles $(b)$ to the penetration depth of sheet piles $(D), b / D$, and the ratio of the radius of circular wall $(R)$ to $D, R / D$, can be added, respectively, for a noexcavation case $(d=0)$. The excavation ratio $d /(D+d)$ should also be added as a parameter for an excavation case $(d>0)$. The values of $b$ and $B(=2 b)$ 

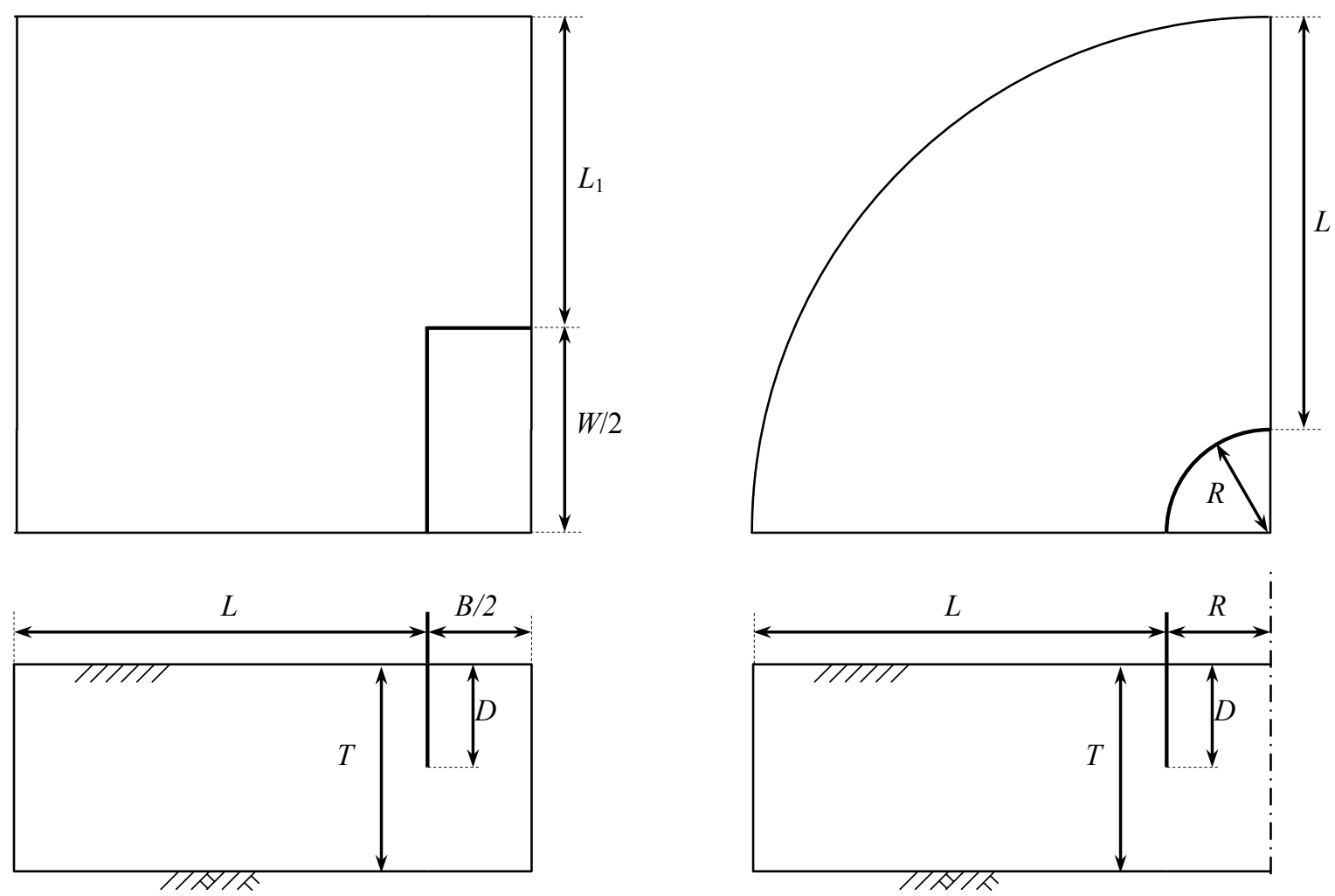

Figure 12. 3D flow (1/4 of a 3D cofferdam)

Figure 13. AXS flow (1/4 of a circular wall)

are the half and total breadth of double sheet pile walls, respectively.

In the case of 3D flow (see Fig. 12), another ratio, $W / B$, relating to the plane shape should be added as well as the ratio of $b / D$, where $B(=2 b)$ and $W(=$ $2 w)$ are lengths of the short and long sides of a rectangle in a plane shape of a 3D cofferdam, respectively.

As shown in Figures 10 to $12, L$ is the distance between the sheet pile wall and the impervious left or right-hand side boundary, which is taken as an infinite boundary.

\subsection{Appropriateness of non-dimensional formulization of $\mathrm{H}_{c}$ for seepage failure of soil}

Let us now discuss whether or not the nondimensional formulization of $H_{c}$ for the seepage failure of soil is appropriate. With respect to the 2D, 2DC, 3D, and AXS flows as shown in Figures 10 to 13, FEM (Finite Element Method) seepage flow and stability against the seepage failure of soil were analyzed for real, 1/50 model, and 1/100 model soils. As an example, the real soil has the sizes of $L=40 \mathrm{~m}, T$ $=20 \mathrm{~m}, D=10 \mathrm{~m}, t=0 \mathrm{~mm}, d=0 \mathrm{~m}$, and $\gamma^{\prime} / \gamma_{w}=$ 1.0 , where $L$ is the distance between the sheet pile wall and the impervious left or right-hand side boundary, $T$ is the total depth of soil, $D$ and $t$ are the penetration length and thickness of the sheet pile wall, respectively, $d$ is the excavation depth downstream, $\gamma_{w}$ is the unit weight of water, and $\gamma^{\prime}$ is the buoyant unit weight of soil. We use the following notation for some flow conditions: $B(=2 b)$ is the breadth of double sheet pile walls under the 2DC flow condition, $R$ is the radius of circular wall in an axisymmetric flow under the AXS flow condition, and $B(=2 b)$ and $W(=2 w)$ are the lengths of short and long sides of the rectangular wall, respectively (in the plane shape) under the 3D flow condition. As an example of real soil, these values have the magnitudes of $B=20 \mathrm{~m}, R=10 \mathrm{~m}$, and $W=40 \mathrm{~m}$. The model soils correspond to the actually tested soils, i.e., the $1 / 50$ models to $2 \mathrm{D}, 2 \mathrm{DC}, 3 \mathrm{D}$, and AXS test soils, and 1/100 models to H2D, H2DC, H3D, and HAXS test soils. Here, for instance, the H2D test means a half model of the 2D seepage failure experiment. The authors have already conducted 2D, 2DC, $3 \mathrm{D}, \mathrm{AXS}$, and $\mathrm{H} 2 \mathrm{D}$ experiments in their laboratory.

Here, the critical hydraulic head differences, $H_{c}$, obtained by the numerical analyses are considered. In the two-dimensional case, analyses were conducted using a Fortran program FEMNESD7 for FEM seepage flow with 4CST elements (Desai and Abel, 1972), and Fortran program SEEPFL57 for the stability against seepage failure based on the pfc $2 \mathrm{D}$. In the axisymmetric case, FENESYD3 for FEM seepage flow with 4CST elements, and SEEPFL57 for stability against seepage failure based on the pfc AXS were used, and in the three-dimensional case, FEMSEE6E for seepage flow with iso-parametric elements composed of 27 nodes, and SEEPFL67 for stability against seepage failure. These programs, not coupled, were coded in the authors' Laboratory.

As for the 2D flow condition, Table 1 (a) shows the values $H_{c}$ and those non-dimensional values $H_{c} \gamma_{w} / T \gamma^{\prime}$, in the cases of the 2D real (prototype), 2D test (1/50 model), and H2D test (1/100 model) soils. Table 1 (a) shows that the $H_{c} \gamma_{w} / T \gamma$ ' values are almost the same for real, $1 / 50$, and 1/100 models. It has been verified that the above theory of the non- 
Table $1 . H_{c}$ and $H \gamma_{w} / T \gamma^{\prime}$ values in the cases of the 2D, 2DC, 3D and AXS flows for the real (prototype), 1/50, and 1/100 model soils

\begin{tabular}{llrrrrrrr}
$H_{c}$ & $\gamma^{\prime}$ & $\gamma_{w}$ & $T$ & & $D$ & $B / 2$ & $W / 2$ & $H_{c} \gamma_{w} / T \gamma^{\prime}$ \\
\hline $\mathrm{m}$ & $\mathrm{kN} / \mathrm{m}^{3}$ & & $\mathrm{kN} / \mathrm{m}^{3}$ & $\mathrm{~m}$ & $\mathrm{~m}$ & $\mathrm{~m}$ & $\mathrm{~m}$ &
\end{tabular}

\begin{tabular}{lllllll}
\hline (a) 2D & \multicolumn{7}{l}{} & \\
\hline 2Dreal & 29.638990 & 9.80000 & 9.80000 & 20.00 & 10.00 & 1.4819495 \\
2Dmodel & 0.5927794 & 9.80000 & 9.80000 & 0.400 & 0.200 & 1.4819485 \\
2Dmodel (B) & 0.5102466 & 8.43555 & 9.80000 & 0.400 & 0.200 & 1.4819481 \\
H2Dmodel & 0.2963904 & 9.80000 & 9.80000 & 0.200 & 0.100 & 1.4819520
\end{tabular}

\begin{tabular}{|c|c|c|c|c|c|c|c|}
\hline \multicolumn{8}{|l|}{ (b) $2 \mathrm{DC}$} \\
\hline 2DCreal & 22.293860 & 9.80000 & 9.80000 & 20.00 & 10.00 & 10.00 & 1.1146930 \\
\hline 2DCmodel & 0.4458785 & 9.80000 & 9.80000 & 0.400 & 0.200 & 0.200 & 1.1146963 \\
\hline 2DCmodel (B) & 0.3837988 & 8.43555 & 9.80000 & 0.400 & 0.200 & 0.200 & 1.1146961 \\
\hline H2DCmodel & 0.2229396 & 9.80000 & 9.80000 & 0.200 & 0.100 & 0.100 & 1.1146980 \\
\hline
\end{tabular}

\begin{tabular}{lcccccccc}
\hline (c) 3D & \multicolumn{10}{c}{ 3D } \\
\hline 3Dreal & 16.315670 & 9.80000 & 9.80000 & 20.00 & 10.00 & 10.00 & 20.00 & 0.8157835 \\
3Dmodel & 0.3263146 & 9.80000 & 9.80000 & 0.400 & 0.200 & 0.200 & 0.400 & 0.8157865 \\
3Dmodel (B) & 0.2808819 & 8.43555 & 9.80000 & 0.400 & 0.200 & 0.200 & 0.400 & 0.8157867 \\
H3Dmodel & 0.1631568 & 9.80000 & 9.80000 & 0.200 & 0.100 & 0.100 & 0.200 & 0.8157840 \\
\hline (d) AXS & & & & & & & & \\
\hline AXSreal & 15.506710 & 9.80000 & 9.80000 & 20.00 & 10.00 & 10.00 & 0.7753355 \\
AXSmodel & 0.3101349 & 9.80000 & 9.80000 & 0.400 & 0.200 & 0.200 & 0.7753373 \\
AXSmodel (B) & 0.2669548 & 8.43555 & 9.80000 & 0.400 & 0.200 & 0.200 & 0.7753372 \\
HAXSmodel & 0.1550672 & 9.80000 & 9.80000 & 0.200 & 0.100 & 0.100 & 0.7753360
\end{tabular}

(B): Biwa Lake sand $3\left(\gamma^{\prime} / \gamma_{w}=0.86077\right)$

dimensional formulization is true, and the nondimensional and normalized value $H_{c} \gamma_{w} / T \gamma^{\prime}$ is appropriate under the 2D flow condition.

For the 2DC, 3D, and AXS flow conditions, Table 1 (b), (c), and (d) show the values $H_{c}$ and those non-dimensional values $H_{c} \gamma_{w} / T \gamma$, in the cases of the prototype, $1 / 50$, and $1 / 100$ models, respectively. Table 1 (b), (c), and (d) show that the $H_{c} \gamma_{w} / T \gamma^{\prime}$ values are almost the same for real, $1 / 50$, and 1/100 models. It has been verified that the above theory of the nondimensional formulization is true, and the nondimensional and normalized value $H_{c} \gamma_{w} / T \gamma^{\prime}$ is also appropriate for the 2DC, 3D, and AXS flow conditions.

To validate a non-dimensional formulation for buoyant unit weight $\gamma$, the analytical results with $\gamma^{\prime} / \gamma_{w}=0.86077$ for $2 \mathrm{D}, 2 \mathrm{DC}, 3 \mathrm{D}$, and AXS flows are shown in Table 1 (a), (b), (c), and (d), respectively. The notation (B) in the left column of Table 1 shows the case where $\gamma^{\prime} / \gamma_{w}=0.86077$ is used in the calculation. With respect to the value of $\gamma^{\prime}$, the above theory of the non-dimensional formulization is true, and the non-dimensional and normalized value $H_{c} \gamma_{w} / T \gamma^{\prime}$ is also appropriate for the 2D, 2DC, 3D, and AXS flow conditions.

The above discussion is restricted to the cases of $t$ $=0$ and $d=0$, where $t$ is the thickness of the sheet pile wall, and $d$ is the excavation depth on the downstream side. However, the same thing is said for the cases where $t$ and/or $d$ has a certain value, as long as we consider the prototype and model soils which are geometrically similar, consisting of the same material (sand), and under the same conditions.

\section{CONCLUSIONS}

In this paper, the prismatic failure concept was first presented, which is one of the conventional methods for designing soil excavation safety against seepage failure. The non-dimensional formulization of the critical hydraulic head difference against seepage failure, $H_{c}$, was discussed especially in two dimensions. In the discussion, the flow conditions such as two-dimensional (2D), two-dimensional concentrated (2DC), three-dimensional (3D), and axisymmetric (AXS) flow conditions were considered. When the prototype (real) and model (test) soils are geometrically similar, we discussed whether or not the nondimensional formulization for $H_{c}$ is possible. The following results were obtained:

(1) Non-dimensional values of $D / T$, and normalized values $H_{c} \gamma_{w} / D \gamma^{\prime}$ and $H_{c} \gamma_{w} / T \gamma^{\prime}$ are the same for real and model soils that are geometrically similar, where $D$ is the penetration depth of the sheet pile wall, $T$ is the total depth of soil, and $\gamma^{\prime}$ and $\gamma_{w}$ are the buoyant unit weight of soil and unit weight of water, respectively.

(2) Even if the buoyant unit weights of prototype and model soils are different, the same nondimensional values $D / T$, and the same nondimensional and normalized values $H_{c} \gamma_{w} / D \gamma^{\prime}$ and $H_{c} \gamma_{w} / T \gamma^{\prime}$ are also obtained.

(3) The non-dimensional equations of $H_{c}$ for the infinite and finite depths of 2D soils are as follows, based on Terzaghi's method: 
$\frac{\gamma_{w} H_{c}}{D \gamma^{\prime}}=\frac{1}{C_{0}}=$ Const.

for soil of an infinite depth, and

$\frac{\gamma_{w} H_{c}}{T \gamma^{\prime}}=\frac{1}{C_{0}} \frac{D}{T}$

for soil of a finite depth in two dimensions, where $C_{0}$ is a value depending on hydraulic, geometric, and soil conditions. $C_{0}$ is a constant for soil of an infinite depth, and is a function of $D / T$ for soil of a finite depth.

(4) Similar relationships are true for 2DC, 3D, and AXS flow conditions. Some non-dimensional parameters showing the shape of a cofferdam should be added to describe the excavation cross-sectional shapes.

(5) Numerical analyses show that the nondimensional and normalized values $H_{c} \gamma_{w} / T \gamma^{\prime}$ are the same in the cases of the prototype, $1 / 50$, and $1 / 100$ models for the 2D, 2DC, 3D, and AXS flow conditions, respectively.

The above discussion is restricted to the cases of $t$ $=0$ and $d=0$. However, the same thing is said for the cases where $t$ and/or $d$ has a certain value, as long as we consider the prototype and model soils which are geometrically similar, consisting of the same material (sand), and under the same conditions.

\section{ACKNOWLEDGEMENTS}

A part of this work was supported by JSPS KAKENHI Grant Number 24380131. We wish to express our deepest thanks to JSPS.

\section{REFERENCES}

Desai, C. S. \& Abel J. F. 1972. Introduction to the Finite Element Method. New York: Van Nostrand Reinhold Company: 102-104 and 125-126.

JRA ed. 1999. Highway Earthwork Series: Manual for Temporary Works. Tokyo: Japan Road Association: 78-82 (in Japanese).

JSSMFE ed. 1975. Design Method for Constructing Soil Retaining Structures, Soil engineering library No.11: 94-97 (in Japanese).

Kälin, M. 1977. Hydraulic piping -theoretical and experimental findings. Canadian Geotechnical Journal 14(1): 107-124.

Miura, K., Supachawarote, C. \& Ikeda, K. 2000. Estimation of three dimensional seepage force inside cofferdam regarding boiling type of failure. Proceedings of the Geotech-Year 2000, Developments in Geotechnical Engineering, Bangkok, 27-30 November 2000: 371-380.

Miura, K., Takaji, K., Furukawa, Y. and Imafuku, M. 1992. Safety analysis of cofferdam for bridge footing construction against boiling failure. Proceedings of the 27th annual meeting of Japanese Society of Soil Mechanics and Foundation Engineering: 2005-2008 (in Japanese).

Nagai, S., Tanaka, T. and Kusumi, S. 2016. Case studies on three-dimensional seepage failure of soil within a cofferdam. Transactions of JSIDRE: in press (in Japanese).
Tanaka, T. 1996. Seepage failure of sand within a cofferdam Prismatic failure concept and analytical consideration-. Transactions of JSIDRE 64(6): 77-87 (in Japanese).

Tanaka, T., Hayashi, K. and Yamada, M. 2000. Seepage failure of soil in an axisymmetric condition. Proceedings of the Geotech-Year 2000, Developments in Geotechnical Engineering, Bangkok, 27-30 November 2000: 665-674.

Tanaka T., Hirose, D. and Kusumi, S. 2012. Seepage failure of sand within a cofferdam in three dimensions -Prismatic failure concept 3D and analytical consideration-. Report of Research Center for Urban Safety and Security, Kobe University (16): 285-299 (in Japaese).

Tanaka, T. Kusaka, T., Nagai, S. and Hirose, D. 2009. Characteristics of seepage failure of soil under various flow conditions, Proceedings of the 19th International Offshore and Polar Engineering Conference \& Exhibition 2009 (ISOPE-2009), Osaka: 90-94.

Tanaka, T. and Verruijt, A. 1999. Seepage failure of sand behind sheet piles - The mechanism and practical approach to analyze-. Soils and Foundations "Underground Construction in Soft Ground" 39(3): 27-35.

Tanaka, T., Verruijt, A. and Hyashi, K. 1999. Seepage failure experiments on sand behind sheet piles. Proceedings of the International Symposium on Geotechnical Aspects of Underground Construction in Soft Ground (IS-Tokyo '99): 717-722.

Terzaghi, K. 1943. Theoretical Soil Mechanic. New York: John Wiley \& Sons: 257-261.

Terzaghi, K. and Peck, R.B. 1948. Soil Mechanics in Engineering Practice, 1 st ed. New York: John Wiley and Sons: 502-503 and 510-512.

Yamaguchi, H. 1976. Soil Mechanics (Lecture and seminar), Revised and enlarged edition, Tokyo: Gihodo: 228 and 265 (in Japanese). 